\title{
The Diagnosis of Choriocarcinoma in Molar Pregnancies: A Revised Approach in Clinical Testing
}

\author{
Lisa Duffy ${ }^{\mathrm{a}}$, Liangtao Zhanga ${ }^{\mathrm{a}}$, Karen Sheath ${ }^{\mathrm{a}}$, Donald R. Love ${ }^{\mathrm{a}}$, Alice M. George ${ }^{\mathrm{a}, \mathrm{b}}$
}

\begin{abstract}
Background: Hydatidiform moles occur in approximately 1 in 1,500 pregnancies; however, early miscarriages or spontaneous abortions may not be correctly identified as molar pregnancies due to poor differentiation of chorionic villi.

Methods: The current clinical testing algorithm used for the detection of hydatidiform moles uses a combination of morphological analysis and 557 immunostaining followed by ploidy testing to establish a diagnosis of either a complete or partial molar pregnancy. We review here 198 referrals for fluorescence in situ hybridization (FISH) ploidy testing, where the initial diagnosis based on morphology is compared to the final diagnosis based on a combination of morphology, FISH and p57 immunohistochemical (IHC) staining.
\end{abstract}

Results: Approximately $40 \%$ of cases were determined to be genetically abnormal, but only $28.8 \%$ of cases were diagnosed as molar pregnancies. The underestimation of complete molar pregnancies and those with androgenetic inheritance was also found to be likely using conventional diagnostic methods, as atypical p57 staining was observed in approximately $10 \%$ of cases.

Conclusions: Our findings suggest that a revised approach to testing products of conception is necessary, with cases screened according to their clinical history in order to distinguish molar pregnancy referrals from hydropic pregnancies.

Keywords: Fluorescence in situ hybridization; Hydatidiform moles; Molar pregnancies; Ploidy

\footnotetext{
Manuscript accepted for publication July 06, 2015

aDiagnostic Genetics, LabPLUS, Auckland City Hospital, Auckland, New Zealand

bCorresponding Author: Alice M. George, Diagnostic Genetics, LabPLUS, Auckland City Hospital, PO Box 110031, Auckland 1148, New Zealand. Email: AliceG@adhb.govt.nz
}

doi: http://dx.doi.org/10.14740/jocmr2236w

\section{Introduction}

Molar pregnancies occur in approximately $1 / 1,500$ pregnancies in western populations [1,2], and are generally detected by the second or third month of pregnancy, either by ultrasound or an elevated human chorionic gonadotropin $(\mathrm{hCg})$ level $[1,3]$. In such pregnancies, an abnormal placental mass develops as a result of a diandric genome (a diploid paternal complement) following an unviable fertilization event [4]. In the case of a complete mole, an empty egg is fertilized by either a single sperm in which the genome has undergone replication, or two individual sperm, causing the absence of a maternal genomic complement (Fig. 1, Table 1) [5]. Complete moles are characterized by grossly swollen villi, giving the placenta an appearance of a bunch of grapes, which can make it hard to differentiate histologically from a hydropic abortus. Hydropic degeneration of villi may also occur following spontaneous abortion of a biparental pregnancy due to abnormal development of villus vasculature [6].

In partial moles, a normal egg is fertilized by either a single sperm in which the genome has undergone replication, or two individual sperm, leading to a triploid genomic complement (Fig. 1, Table 1). The presence of a maternal complement means that only some of the villi show swelling, leading to two morphologically different populations within the same sample. The villi also show characteristic features such as scalloping, cistern formation and trophoblastic proliferation, and are thus easier to detect than complete moles. These characteristic features are less identifiable in early pregnancy loss, however, as villi earlier than 12 weeks gestation may not show typical features of a molar pregnancy and can therefore be hard to diagnose [5]. The best results are achieved when p57 immunohistochemical (IHC) staining and fluorescence in situ hybridization (FISH) ploidy testing are performed in conjunction with morphological analysis (Table 1).

The $\mathrm{p} 57^{\mathrm{KIP} 2} \mathrm{IHC}$ stain is a nuclear stain that is used to detect diandry. The $p 57$ gene is maternally expressed and paternally methylated [7]. In a normal pregnancy with biparental inheritance, the p57 stain would be positive due to the presence of a maternal genetic complement, whereas a diandric complete molar pregnancy would show absence of staining due to the epigenetic silencing of the $p 57$ gene. p57 staining is usually performed in conjunction with FISH ploidy testing in order to detect triploid partial molar pregnancies. Such pregnancies 
COMPLETE MOLE (DIPLOID)

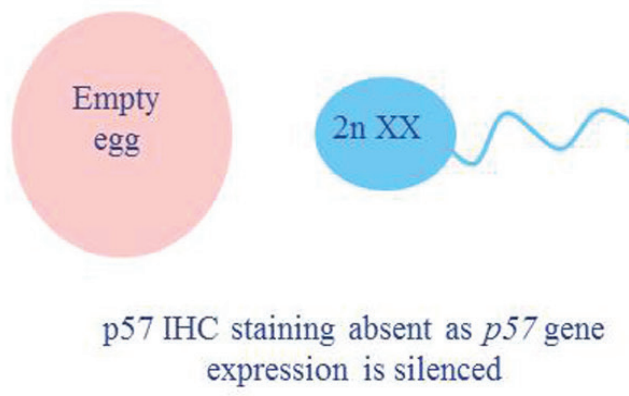

PARTIAL MOLE (TRIPLOID)
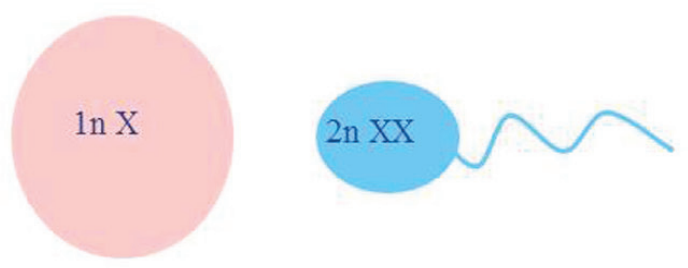

p $57 \mathrm{IHC}$ staining is present as the $p 57$ gene

is expressed

Figure 1. A comparison of the typical ploidy and p57 status in molar pregnancies.

would still show positive $\mathrm{p} 57$ staining due to the presence of a maternal genomic complement, so the assessment of ploidy is required to avoid being scored as a diploid pregnancy.

The identification and classification of molar pregnancies is desirable due to a $15 \%$ risk of choriocarcinoma development in the case of a complete mole, compared to a $0.5 \%$ risk for a partial mole [4]. A choriocarcinoma occurs when cells from the abnormal placental mass become cancerous and metastasize to other parts of the body such as the uterus, lungs or brain [1]. The $p 57$ gene is a candidate tumor suppressor gene, therefore silencing of the gene in a molar pregnancy leads to either functional nullisomy or monosomy, and the potential expression of other recessive oncogenes. Without the detection and successful treatment of a complete mole, subsequent pregnancy attempts can cause symptoms to worsen, leading to TB-like symptoms if the lungs are infiltrated, or headaches, dizziness and seizures if the brain is infiltrated [1]. Treatment is generally successful in greater than $90 \%$ of cases, so early detection is desirable [1].

In this study, we analyzed both the clinical referral reasons and the results of combined morphological analysis, FISH and p57 staining in order to estimate the frequency and detection rate of molar pregnancies, and to assess whether such an approach provides an effective detection strategy for hydatidi- form moles and choriocarcinomas.

\section{Methods}

One hundred and ninety-eight molar pregnancy FISH testing referrals were reviewed, with pathology reports evaluated before and after FISH testing to assess the accuracy of diagnosis by morphology. A second analysis was also performed on 104 cases in which the p57 IHC staining result was available, thereby allowing an estimate of the level of concordance between FISH results and $p 57$ gene expression.

Two FISH slides were prepared for each case by first aging the slides for $20 \mathrm{~min}$ in a $60{ }^{\circ} \mathrm{C}$ oven, then placing them in xylene for 15 min to remove the wax. They were next placed in a $100 \%, 80 \%$, and $70 \%$ ethanol series for 2 min each, ending with $2 \mathrm{~min}$ in deionized water at room temperature. Slides were drained briefly, $30-60 \mu \mathrm{L}$ of $0.2 \mathrm{M} \mathrm{HCl}$ solution was pipetted on to each of the slides, and then they were covered with parafilm and incubated in a humidity chamber at $37^{\circ} \mathrm{C}$ for $20 \mathrm{~min}$. Following this, slides were rinsed in a coplin jar of deionized water and drained, then $30-60 \mu \mathrm{L}$ of heat pretreatment solution (SPoT-Light Tissue Pretreatment Kit) was pipetted onto the slides. They were then covered with a glass coverslip, and sealed

Table 1. A Comparison of the Characteristics of Partial and Complete Moles (Derived From [5])

\begin{tabular}{lll}
\hline Characteristic & Partial mole & Complete mole \\
\hline Development & Some fetal development but malformed & No fetal development (cord, membrane present) \\
Villi & $\begin{array}{l}\text { Some enlarged; two populations of villi } \\
\text { Blood vessels and fetal red blood cells present } \\
\text { Scalloped outlines with pseudoinclusions and invaginations }\end{array}$ & All enlarged \\
& $\begin{array}{l}\text { Focal trophoblast proliferation } \\
\text { Minimal trophoblast atypia }\end{array}$ & $\begin{array}{l}\text { Round to ovoid } \\
\text { Prominent cisterns }\end{array}$ \\
& & Circumferential trophoblastic proliferation \\
& Both maternal and paternal & Trophoblast atypia present \\
\hline Origin & Triploid & Paternal only \\
Genetics & Positive & Diploid (or tetraploid) \\
p57 IHC stain & & Negative \\
\hline
\end{tabular}


Table 2. Referral Reasons for Ploidy Testing Based on Morphology Compared to the Final Diagnosis Using FISH

\begin{tabular}{|c|c|c|c|c|}
\hline & \multicolumn{4}{|c|}{ Initial referral reason } \\
\hline & $\begin{array}{l}\text { Molar pregnancy } \\
\text { referral }(n=70)\end{array}$ & $\begin{array}{l}\text { Exclude molar pregnancy } \\
\text { referral }(n=39)\end{array}$ & $\begin{array}{l}\text { Hydropic/Other } \\
\text { referral }(n=89)\end{array}$ & Total $(n=198)$ \\
\hline \multicolumn{5}{|l|}{ Final diagnosis } \\
\hline Molar pregnancy & 40 & 5 & 12 & 57 \\
\hline Hydropic pregnancy & 23 & 30 & 69 & 122 \\
\hline Percentage correctly diagnosed (\%) & $57.1 \%$ & $87.2 \%$ & $86.5 \%$ & \\
\hline
\end{tabular}

with rubber cement. Slides were heated on the thermal cycler for 30 min at $95^{\circ} \mathrm{C}$ and then the coverslip was gently removed and the slides immersed in deionized water. After draining briefly, 15 - $30 \mu \mathrm{L}$ of enzyme reagent (SPoT-Light Tissue Pretreatment Kit) was added to the slides, they were covered with a square of parafilm, and incubated for $30 \mathrm{~min}$ in a humidified chamber at $37^{\circ} \mathrm{C}$. The parafilm was then removed, the slides washed briefly in a coplin jar of deionized water at room temperature, and finally dehydrated for $2-3$ min each in each of $70 \%, 80 \%$ and $100 \%$ ethanol solution and air dried at room temperature.

The pretreated paraffin slides were matched against a corresponding marked H\&E slide (Shandon Rapid-Chrome ${ }^{\mathrm{TM}}$ Frozen Section Staining kit), and the area for testing transferred to the pretreated slides using a marker pen initially, followed by a diamond-tipped engraver. The XY18 and 13,21 Vysis aneuscreen probe sets (Abbott Molecular) were then applied to the hybridization site marked on each slide, a glass coverslip applied, and the area was sealed with rubber cement. The slides were denatured in the thermal cycler slide for $20 \mathrm{~min}$ at 85 ${ }^{\circ} \mathrm{C}$, and then placed in a humidified box in the incubator at 37 ${ }^{\circ} \mathrm{C}$ for $12-16 \mathrm{~h}$. A post hybridization wash was performed by gently removing the rubber cement, and then washing slides in a coplin jar of $0.4 \times \mathrm{SSC} / 0.03 \% \mathrm{NP} 40$ solution at $72{ }^{\circ} \mathrm{C}$ for $2 \mathrm{~min}$, before being transferred to $2 \times \mathrm{SSC} / 0.01 \% \mathrm{NP} 40$ at room temperature for $30 \mathrm{~s}$. Slides were then drained, Vectashield with DAPI and a coverslip applied, and finally visualized under a fluorescence microscope. Slides were analyzed by two observers, each analyzing a minimum of four different areas within the targeted area.

\section{Results}

Prior to FISH testing, pathology referrals fell into three main groups (Table 2): those querying a molar pregnancy (70/198), those wanting to exclude a molar pregnancy (39/198), and those that queried a hydropic pregnancy (89/198). After FISH testing, however, 57/198 (28.8\%) were diagnosed as molar pregnancies, with the majority $(122 / 198$ or $61.6 \%)$ classed as hydropic (Table 2). This result shows that the diagnosis of molar pregnancies by morphology is less accurate than the diagnosis of hydropic pregnancies (Table 2).

In total, $82 / 198(41.4 \%)$ cases were determined to be genetically abnormal, either as a molar pregnancy or an aneuploid conceptus. Among the 82 abnormal cases, 4.5\% (9/198) were classified as complete moles and 24.2\% (48/198) were classed as partial moles, with the remaining $25 / 198$ (12.6\%) cases reported as aneuploid. The aneuploidy cases were trisomy 13 (six cases), trisomy 18 (seven cases), trisomy 21 (four cases), monosomy 21 (two cases), monosomy $\mathrm{X}$ (five cases) and a double trisomy for chromosomes 13 and 18 .

The ploidy results were generally concordant with the p57 IHC staining, although atypical p57 staining was observed in $10 / 104(9.6 \%)$ cases overall (Table 3$)$. When these atypical cases were examined, 8/10 showed an absence of p57 IHC staining (Table 3), indicating androgenetic inheritance. Despite this, the

Table 3. A Summary of the Cases Showing Atypical p57 IHC Staining Patterns

\begin{tabular}{lllll}
\hline Category & P57 stain & Sex & Other abnormality? & Maternal genome status and possible causal mechanism \\
\hline Complete mole & Positive & XX & No & Present - retained maternal chromosome 11/trisomic rescue \\
Partial mole & Negative & XXY & Triploid & Absent - dispermy or mutation in maternal p57 allele \\
& Negative & XXX & Triploid & Absent - dispermy or mutation in maternal p57 allele \\
& Positive & XX & No & Present - biparental mole \\
& Negative & XXY & Triploid & Absent - dispermy or mutation in maternal p57 allele \\
Hydropic & Negative & XXY & Triploid & Absent - dispermy or mutation in maternal p57 allele \\
& Negative & XY & T18 & Absent - dispermy, biparental mole or mutation in maternal p57 allele \\
& Negative & XX & T21 & Absent - dispermy, biparental mole or mutation in maternal p57 allele \\
\hline Other & Negative & XY & No & Absent - dispermy, biparental mole or mutation in maternal p57 allele \\
\hline & Negative & XX & No & Absent - dispermy, biparental mole or mutation in maternal p57 allele \\
\hline
\end{tabular}


Table 4. A Comparison of the Sex Complement and p57 IHC Staining Results in Molar Pregnancies

\begin{tabular}{llll}
\hline Category & Sex complement & Number of cases & p57 stain \\
\hline Complete mole (p57-) & XX & 8 & $\begin{array}{l}\text { One case atypical (p57+): focally positive staining in } \\
\text { the cytotrophoblast }\end{array}$ \\
& XY & 0 & \\
Partial mole (p57+) & XXY & 14 & $\begin{array}{l}\text { Three cases atypical: two were p57 negative, and one } \\
\text { showed absent staining in the basal cytotrophoblast }\end{array}$ \\
& XYY & 1 & Suboptimal \\
XXX & 3 & One case atypical: p57 negative \\
Total & 1 & One case: p57 positive \\
\hline
\end{tabular}

final diagnosis was made based on the ploidy level of the sample, rather than inheritance pattern in $9 / 10$ cases (Table 3 ), so the percentage of cases with androgenetic inheritance is likely to be underestimated. The risk of underestimation due to discordance between the IHC and ploidy increased when only the cases diagnosed as molar pregnancies were assessed. In total, $27 / 104(26.0 \%)$ cases were diagnosed as molar pregnancies, but out of 26 which showed definitive p57 staining, 6/27 (22.2\%) of these cases showed discordance between the IHC and FISH results (Table 4). Closer examination showed that three of the atypical cases had atypical staining in the cytotrophoblast (Table 4), suggesting the presence of different cell lines.

\section{Discussion}

The results reported here highlight a need for the clear dif- ferentiation of genetic disorders that result in fetal demise, and those that are detrimental to the mother. Fetal demise may occur for a number of reasons, but molar pregnancies put the health of the mother at risk, and therefore need to be accurately diagnosed and treated. Approximately $10-15 \%$ of molar pregnancies are invasive, causing hemorrhaging and other complications, with 2-3\% developing into a choriocarcinoma $[1,4]$. Diagnosis can be difficult, particularly in early pregnancy loss, when many of the characteristic features of molar pregnancies are ill-defined in the villi due to poor tissue differentiation [5].

The analysis of the initial referral reasons for FISH ploidy testing showed that the majority of referrals $(60.6 \%)$ used FISH testing as an exclusion tool, rather than for a definitive diagnosis of the disease (Table 2). As $44.9 \%$ of samples in this study were querying hydropic villi rather than molar pregnancies at the outset, and $71.2 \%$ of pregnancies were later found

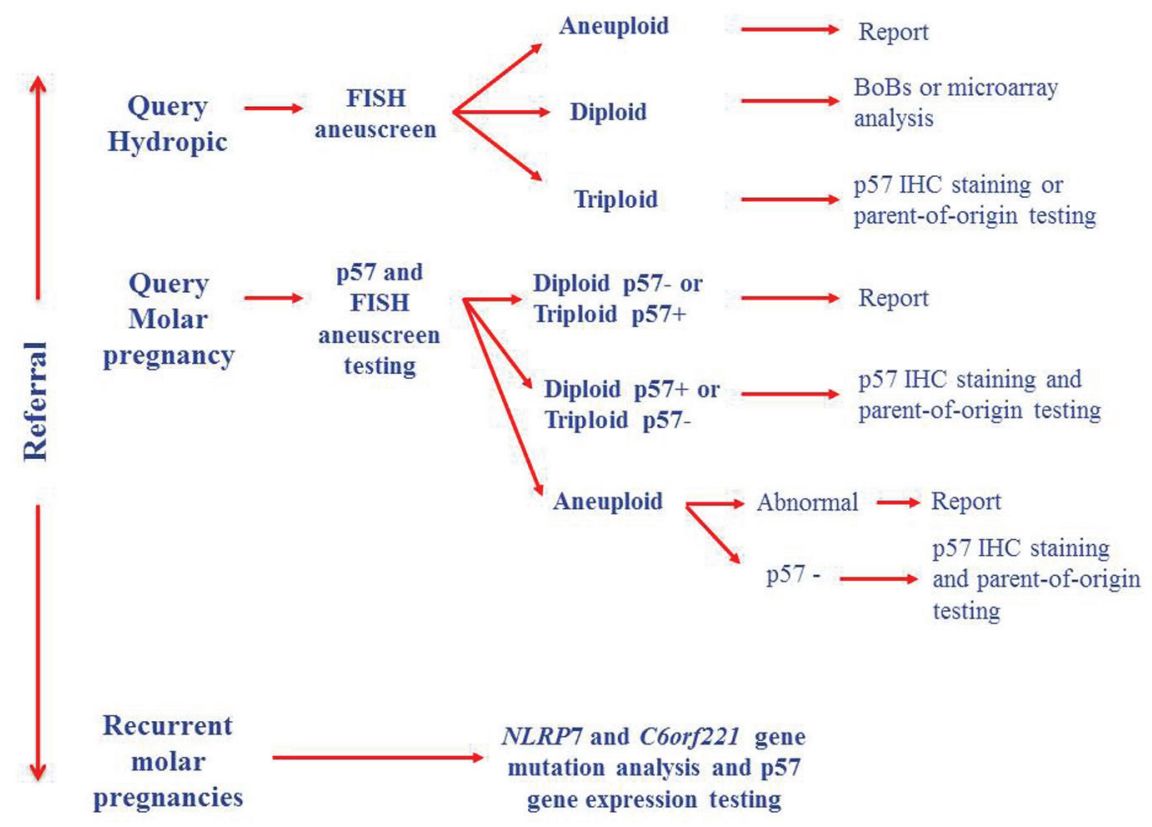

Figure 2. Proposed testing algorithm for products of conception. 
to be hydropic or non-molar, an alternative method of genetic testing may have been more appropriate at the outset.

BACs-on-Beads (BoBs) and microarray technologies [810] have been successfully used in a number of centers for the testing of products of conception, thus increasing the detection rate of genetic abnormalities. These techniques could be combined with genotyping [11] and mutational analysis in apparently normal cases to test for inheritance disorders (Fig. 2). Hydatidiform moles are considered to be a disorder of genomic imprinting [12], therefore genotyping on its own is insufficient for the diagnosis of molar pregnancies [12], and may be of limited use if DNA quality is poor or maternal contamination is greater than $20 \%$ [11].

Current testing methods are likely to underestimate the frequency of both complete moles and pregnancies with diandric inheritance. In this study, complete moles had the lowest detection rate, which could either reflect the rarity of such an event, or inadequacy of the p57 stain as a definitive test for androgenetic inheritance. Approximately $10 \%$ of referrals in this study showed atypical p57 staining patterns (Table 3 ), and the majority showed loss of expression, suggesting either the complete absence of a maternal genome in the fetus, or that loss of the p57 function had occurred via an alternative mechanism. The absence of p57 staining occurred in four cases diagnosed as hydropic abortuses, which may in fact have been complete moles due to either uniparental disomy (UPD) for chromosome 11 , or a mutation in the maternal copy of the $p 57$ gene (Table 3). The absence of staining was also observed in four molar pregnancy cases (Table 3), all of which were triploid partial moles (Table 3), suggesting loss of the maternal genomic complement from either a p57 mutation or dispermy. The latter would be unsurprising, as Zaragoza et al [13] reported that approximately two-thirds of triploids are of paternal origin due to dispermy, with a minority $(8.3 \%)$ due to fertilization by a diploid sperm $[4,14]$.

Without molecular genetic studies, it is not possible to determine the absence of p57 staining. This absence may be due to diandric inheritance, UPD for chromosome 11 , or to biparental inheritance with a mutation in the maternal p57 $(C D K N 1 C)$ gene. The $p 57$ gene is located in the $11 \mathrm{p} 15 \mathrm{re}-$ gion, and mutations of the maternal 11p15.5 p57 gene (OMIM 600856) are associated with Silver-Russell syndrome (SRS). Mutations associated with SRS are most frequently caused by loss of methylation in the differentially methylated region 1 (DMR1) at $11 \mathrm{p} 15(50 \%)$, and mutations or aberrant methylation at the maternal chromosome $11 \mathrm{p} 15$ locus are linked to growth retardation, hemihypoplasia, and an increased association with cancer, macrosomia and midline abdominal wall defects, and several other childhood tumors [4], such as Wilms' tumor (OMIM \#194071), adrenocortical carcinoma (OMIM \#202300), and rhabdomyosarcoma (OMIM \#268210). This may explain the increased risk of choriocarcinoma in complete molar pregnancies compared to those with digenic inheritance or paternal UPD of chromosome 11, which causes the opposite overgrowth disorder, Beckwith-Wiedemann syndrome (BWS).

Establishing the parent-of-origin of gene expression imbalance is therefore of importance in the diagnosis of molar pregnancies in order to discern the risk of choriocarcinoma development. A complete mole with p 57 positivity was observed in this study (Table 3), suggesting either retention of the maternal chromosome 11 [15], or a biparental complete mole. Biparental complete moles are generally the result of abnormal methylation of maternal alleles [16] and usually show complete lack of p57 staining [12], although maternal homozygous or compound heterozygous mutations in the $N L R P 7$ and C6orf221 (KHDC3L) genes have also been implicated when fetuses are shown to be diploid and biparental in origin [1719]. Both the 6q13 C6orf221 (OMIM \#614293) and 19q13.42 NLRP7 (OMIM \#609661) genes are expressed in oocytes, and are thought to be involved in setting and/or maintaining the maternal imprint [19], with mutations in the latter thought to be linked to early cleavage errors and subsequent reduced maternal immune response that allows abnormal cells to persist [18]. Maternal imprinting defects or non-synonymous or stop mutations in the NLPR7 gene [2, 12, 18] have also been suggested as the cause of recurrent molar pregnancies. Therefore testing methods that are able to establish parental inheritance and also distinguish between gene mutations or epimutations are desirable, as these would provide an estimate of both the risk of choriocarcinoma development and the recurrence risk of such pregnancies. This would be particularly relevant to fertility or recurrent miscarriage referrals, as diandric triploids are the most frequent chromosome abnormality in infertile males with oligo-, crypto- and azoospermia [14].

The clinical history of the patient should therefore be used to guide testing options (Fig. 2), as patients with a history of molar pregnancies and young recurrent miscarriage couples should be tested at the outset for mutations in the NLRP7 and C6orf 221 genes, and $p 57$ gene expression levels, in order to establish whether mutations or epimutations in these genes are occurring. This would provide information about recurrence risk for future pregnancies, and the risk of choriocarcinoma due to imprinting defects. Cases with no prior clinical history and a morphological diagnosis of a molar pregnancy would be better suited to initial screening by p57 staining and FISH ploidy testing, with atypical cases followed up by parent-oforigin testing to establish whether uniparental disomy has occurred, and also methylation analysis of the $p 57$ gene to assess the risk of choriocarcinoma development. BoBs or microarray testing methods are more appropriate for the detection of aneuploidy or sequence variants in all other cases, combined with p57 IHC staining to provide an indicator of imprinting defects that could be followed up by other methods.

Present testing methods such as ploidy and p57 staining may therefore both underestimate the incidence of complete molar pregnancies and also the complexity of androgenetic inheritance. These methods rely on either the absence of expression of the maternally expressed $p 57$ gene, or the inference of $p 57$ gene expression from the combined assessment of the sex complement of the fetus and the villus morphology. Although they can provide an effective initial screen for molar pregnancies, they should not be used for general early pregnancy losses which are more likely to be due to aneuploid errors. Cases showing discordant results should be followed up by combined parent-of-origin and methylation studies. A revised approach to testing would provide more accurate diagnosis of molar pregnancies, and also allow an estimate of the recurrence risk for couples. 


\section{Conflict of Interests}

The authors declare that they have no conflict of interests.

\section{References}

1. Lurain JR. Gestational trophoblastic disease I: epidemiology, pathology, clinical presentation and diagnosis of gestational trophoblastic disease, and management of hydatidiform mole. Am J Obstet Gynecol. 2010;203(6):531539.

2. Slim R, Mehio A. The genetics of hydatidiform moles: new lights on an ancient disease. Clin Genet. 2007;71(1):2534.

3. Hsieh CC, Hsieh TT, Hsueh C, Kuo DM, Lo LM, Hung TH. Delivery of a severely anaemic fetus after partial molar pregnancy: clinical and ultrasonographic findings. Hum Reprod. 1999;14(4):1122-1126.

4. Gardner RJM, Sutherland GR, Schaffer LG. Chromosome Abnormalities and Genetic Counseling. Book Chapters. 4th Edition. New York: Oxford University Press Inc, 2011.

5. Cheung AN-Y. Gestational trophoblastic disease, Chapter 32. In: Robboy SJ, Mutter GL, Prat J, Bentley RC, Russell P, Anderson MC, eds. Robboy's Pathology of the Female Reproductive Tract. 2nd ed. Churchill Livingstone: Elsevier, Philadelphia, 2008.

6. Jauniaux E. Differential diagnosis and management of molar pregnancy, Chapter 7. In: Jurkovic D, Farquharson R, eds. Acute Gynaecology and Early Pregnancy. London: Royal College of Obstetricians and Gynaecologists (RCOG) Press, 2011:73-84.

7. Pateras IS, Apostolopoulou K, Niforou K, Kotsinas A, Gorgoulis VG. p57KIP2: "Kip"ing the cell under control. Mol Cancer Res. 2009;7(12):1902-1919.

8. Paxton CN, Brothman AR, Geiersbach KB. Rapid aneusomy detection in products of conception using the KaryoLite BACs-on-Beads assay. Prenat Diagn. 2013;33(1):2531.

9. Sheath KL, Duffy L, Asquith P, Love DR, George AM. Bacterial artificial chromosomes (BACs)-on-Beads as a diagnostic platform for the rapid aneuploidy screening of products of conception. Mol Med Rep. 2013;8(2):650654.
10. Gao J, Liu C, Yao F, Hao N, Zhou J, Zhou Q, Zhang $\mathrm{L}$, et al. Array-based comparative genomic hybridization is more informative than conventional karyotyping and fluorescence in situ hybridization in the analysis of first-trimester spontaneous abortion. Mol Cytogenet. 2012;5(1):33.

11. Murphy KM, McConnell TG, Hafez MJ, Vang R, Ronnett BM. Molecular genotyping of hydatidiform moles: analytic validation of a multiplex short tandem repeat assay. J Mol Diagn. 2009;11(6):598-605.

12. Fisher RA, Hodges MD, Rees HC, Sebire NJ, Seckl MJ, Newlands ES, Genest DR, et al. The maternally transcribed gene p57KIP2 (CDNK1C) is abnormally expressed in both androgenetic and biparental complete hydatidiform moles. Human Molecular Genetics. 2002;11(2):3667-3672.

13. Zaragoza MV, Surti U, Redline RW, Millie E, Chakravarti A, Hassold TJ. Parental origin and phenotype of triploidy in spontaneous abortions: predominance of diandry and association with the partial hydatidiform mole. Am J Hum Genet. 2000;66(6):1807-1820.

14. Egozcue S, Blanco J, Vidal F, Egozcue J. Diploid sperm and the origin of triploidy. Hum Reprod. 2002;17(1):5-7.

15. McConnell TG, Norris-Kirby A, Hagenkord JM, Ronnett BM, Murphy KM. Complete hydatidiform mole with retained maternal chromosomes 6 and 11. Am J Surg Pathol. 2009;33(9):1409-1415.

16. El-Maarri O, Seoud M, Riviere JB, Oldenburg J, Walter J, Rouleau G, Slim R. Patients with familial biparental hydatidiform moles have normal methylation at imprinted genes. Eur J Hum Genet. 2005;13(4):486-490.

17. Brown L, Mount S, Reddy R, Slim R, Wong C, Jobanputra $\mathrm{V}$, Clifford P, et al. Recurrent pregnancy loss in a woman with NLRP7 mutation: not all molar pregnancies can be easily classified as either "partial" or "complete" hydatidiform moles. Int J Gynecol Pathol. 2013;32(4):399-405.

18. Deveault C, Qian JH, Chebaro W, Ao A, Gilbert L, Mehio A, Khan R, et al. NLRP7 mutations in women with diploid androgenetic and triploid moles: a proposed mechanism for mole formation. Hum Mol Genet. 2009;18(5):888897.

19. Fallahian M, Sebire NJ, Savage PM, Seckl MJ, Fisher RA. Mutations in NLRP7 and KHDC3L confer a complete hydatidiform mole phenotype on digynic triploid conceptions. Hum Mutat. 2013;34(2):301-308. 